\title{
Augmented bladders and urinary diversions
}

\author{
Peter Metcalfe, $M D^{\prime}$; Shuba De, $M D^{\prime}$; Greg Bailly, $M D^{2}$ \\ 'University of Alberta, Edmonton, AB; ${ }^{2}$ Dalhousie University, Halifax, NS; Canada
}

Cite as: Can Urol Assoc J 2018;12(4Suppl1):S24-6. http://dx.doi.org/10.5489/cuaj.5226

\section{Introduction}

Spina bifida and all of its variants (lipomeningocele, tethered spinal cord) are major congenital anomalies involving multiple organ systems. Most tertiary pediatric centres follow these patients in multidisciplinary dedicated spina bifida clinics involving urology, neurosurgery, orthopedic surgery, physical medicine, general pediatrics, physiotherapy, psychology, and social work. This comprehensive care involves the entire family and is extremely successful in minimizing morbidity and maximizing the potential for quality of life. The nurses and administrators are extremely diligent in ensuring followup and addressing their entire spectrum of concerns.

\section{Caring for patients}

The role of the urologist in the care of these patients is several-fold: to maximize renal health, promote continence, and ensure the long-term health of the kidneys and bladder.

Hydronephrosis and renal failure were common before the advent of $\mathrm{CIC},{ }^{1}$ anticholinergic medications, ${ }^{2}$ and surgical techniques of urinary diversion. ${ }^{3}$ Our pediatric clinic aggressively monitors renal health with annual ultrasounds and stratifies risk with annual urodynamics. CIC is often started in the neonatal period, and antimuscarnics added to maintain detrusor pressures below $20-30 \mathrm{mmHg}$. If this initial management fails, a second antimuscarinic or a beta 3-agonist is added. Intravesical onabotulinumtoxinA can be considered for refractory cases of obstructive uropathy resulting from high detrusor leak point pressures. Further deterioration leading to upper tract uropathy usually requires surgical intervention with a continent diversion (bladder augmentation) for those families considered reliable enough to undertake the significant workload required to care for a complex reconstruction. For those families unwilling or unable to accept the responsibility, an incontinent diversion, such as a vesicostomy in the infant or ileovesicostomy in the older child, is required.

Continence becomes much more of a concern and is addressed in the early school years. Most patients are managed with an aggressive $\mathrm{CIC}$ interval and/or tailoring of

their oral medications. More aggressive measures are often required, and treatment is based upon urodynamic and clinical findings. If the outlet requires a minor to moderate increase in resistance, the suburethral slings using autologous fascia can be employed. Persistent detrusor overactivity may respond well to onabotulinumtoxinA, but in children this usually requires a general anesthetic and, therefore, repeated administration can be a burden.

The most aggressive surgical interventions include a bladder augmentation incorporating a continent catheterizable channel (Mitrofanoff or Monti), and an associated bladder neck reconstruction. A MACE is very successful in managing fecal continence refractory to medical management. These procedures can be very successful at reducing bladder pressures and creating continence, however, the burden of work placed on the patient and family should not be underestimated. ${ }^{4}$ If strict adherence to the catheterizing schedule is not adhered to, continence will never be achieved and the risk of complications is very high. ${ }^{5}$ Although benefits to renal function are relatively easy to demonstrate in the short-term, long-term renal health with enterocystoplasty may be questioned. The quality of life benefits are anecdotally obvious, but difficult to prove in childhood, ${ }^{6}$ and much more complicated to assess and follow in adulthood.?

Continent reconstruction in the spina bifida patient is a major surgical undertaking, and more importantly, requires lifetime followup and diligent care by the patient. An enterocystoplasty will predispose the patient to many minor, significant, and potentially fatal complications. The lifetime risk of needing at least one additional surgery is at least $33 \% .^{5}$ The urologist who commits to the long-term followup and management of the patient with a continent diversion must be familiar with the complications that may arise. Adverse outcomes include:

- Potentially fatal

- Malignancy

- Bladder perforation

- Significant surgical complications

- Bowel obstruction

- Bladder stones

- Stomal and channel stenosis, difficulty catheterizing, or incontinence

- Need for re-augmentation 


\section{Case}

S.C. was born with a lumbar myelomeningocele. He was prescribed clean intermittent catheterizations (CIC), anticholinergics, and a bowel routine starting at the age of three years. Due to a failure of conservative management, with persistent incontinence and elevated detrusor pressures with hydronephrosis, he underwent a Malone antegrade continence enema (MACE), Monti catheterizable channel, and an enterocystoplasty (bladder augmentation with ileum) at the age of 10 years. At the time of surgery, S.C. was living at home, under the care of a stable family, and there had been no evidence of poor compliance with medical recommendations.

S.C. did well after his surgery and was continent. The family ensured regular catheterizing every four hours while awake, and a daily MACE flush. He was followed though our pediatric and then adolescent spina bifida clinics for approximately three years.

Unfortunately, S.C. and his family failed to return to clinic; the family stated that they had moved, making travel less convenient.

S.C. presented to the emergency department at the age of 19 years after 24 hours of diffuse abdominal pain, with leukopenia and in septic shock. He was intubated in the emergency room due to respiratory distress and brought to the operating room (OR) for an emergent laparotomy. Over $2 \mathrm{~L}$ of urine were found in the abdomen and a perforation was identified in the posterior bladder wall at the anastomosis. The patient improved significantly over the next 24 hours, and was extubated and off vasopressors.

Unfortunately, his abdominal pain increased on POD \#4 and required percutaneous drainage of multiple fluid collections. His clinical picture did not improve further, and he was brought back to the OR for a repeat laparotomy. The pediatric urologist responsible for the primary surgery was able to attend and assist. Further fluid collections were drained and suprapubic tube and JP tube placed. The patient recovered well.

Postoperatively, the patient was seen by his adult urologist and in the Adult Spina Bifida Clinic, also attended by his pediatric urologist. However, he subsequently was lost to followup again, having not been seen for over one year.

He eventually returned to out Adult Spina Bifida Clinic, as a referral from the emergency room with continuous incontinence, recurrent infections, and a large bladder stone. He had very transient living conditions and was not catheterizing. The clinic social worker was able to restore some of his benefits and explore assisted living residencies. The urology team discussed further medical and surgical options. Due to concerns of substance abuse, we decided that he was unlikely to reliably catheterize and decided upon an incontinent diversion.

He subsequently underwent an uncomplicated ileovesicostomy and has intermittently returned to clinic for followup. He is happy with his ostomy, and home care has been able to assist with appliance changes.

- Medical complications

- Increased risk of urinary tract infection (UTI)

- Vitamin A, D,E, K deficiency

- Vitamin B12 deficiency

- Diarrhea

These patients require at least an annual followup with clinical assessment, ultrasound, and possibly urodynamics. ${ }^{8}$ Although mortality from malignancy has generated significant interest, screening has not proven effective, and the incidence may be lower than once thought. ${ }^{9,10}$

Although most major Canadian centres have well-established pediatric spina bifida clinics, with comprehensive multidisciplinary care, the transition to adulthood can be very difficult. ${ }^{8}$ The patient, in the absence of a dedicated adult spina bifida clinic, requires a urologist with an interest in neurogenic bladder (with or without enterocystoplasty), a neurosurgeon, and a physical medicine specialist. Other important allied workers include: social worker, dedicated nursing staff, orthotist, and physiotherapist. In Edmonton, we have benefited greatly from a pulmonologist, as the incidence of sleep apnea and restrictive lung disease is extremely high.

Despite the establishment of multidisciplinary care programs, patient compliance can be challenging. Although many remain living at home, a significant number live independently and may not be reliable enough to seek appropriate care. Even those living at home can be difficult to follow, as their parents age and are no longer able to maintain the high levels of care necessary for these patients. Therefore, a dedicated clinical program can be invaluable to ensure that patient contact and followup is maintained.

Acute complications, such as bladder perforation or urosepsis, are usually managed by the on-call urologist, but 
most other complications should be managed by a urologist with experience in neurogenic bladder. The most challenging complications often involve the catheterizable stoma and channel, including urinary incontinence and stomal and limb stenosis leading to difficult catheterization. These problems usually require reconstructing a new channel and sometimes revising the entire augment if capacity and compliance are deficient. Body habitus and obesity are often challenging factors in this patient population. Revision surgery should be performed at a centre of excellence by a urologist with experience in reconstruction.

In patients who develop complications secondary to poor patient compliance of bladder management, the conversion to an incontinent form of diversion, such as an ileal conduit or ileovesicostomy, can be considered. This is a particularly important consideration in those patients who fail further revision of catheterizable channels and those who do not commit to the appropriate self-management of their urinary tract. Ileovesicostomy avoids having to perform cystectomy and altering the native ureteral position. The disadvantages include the potential for recurrent UTI and bladder stones from urinary stasis, although this is not always the case.

\section{Conclusion}

Patients and their care team, approaching the age of transitioning to adult care must be made aware of these challenges and a followup plan developed. One of the biggest challenges can be finding a primary care physician willing and able to undertake the complex needs of these patients. So few adult practitioners are involved in the original pediatric care that inheriting an adult patient can be overwhelming; many caregivers will not be willing, able, or prepared for the task. Transfer to an appropriate adult urologist can be straightforward if there is a dedicated regional surgeon with an interest; however, this can be a daunting task, as these patients can be very labour-intensive, and without the additional support staff inherent to a multidisciplinary clinic, can be overwhelming.

Competing interests: The authors report no competing personal or financial conflicts related to this work.

This paper has been peer reviewed.

\section{References}

1. Lapides J, Lapides J, Diokno AC, et al. Clean, intermittent self-catheterization in the treatment of urinary tract disease. J Urol 1972;107:458-61. https://doi.org/10.1016/S0022-5347(17)61055-3

2. Kaefer $M$, Kaefer $M$, Pabby A, et al. Improved bladder function after prophylactic treatment of the high risk neurogenic bladder in newborns with myelomentingocele. J Urol 1999;162:1068-71.

3. Biers SM, Venn SN, Greenwell TJ. The past, present, and future of augmentation cystoplasty. BJU Int 2012;109:1280-93. https://doi.org/10.1111/i.1464-410X.2011.10650.x

4. Clark T, Pope JC, Adams MC, et al. Factors that influence outcomes of the Mitrofanoff and Malone antegrade continence enema reconstructive procedures in children. J Urol 2002;168:1537-40. https://doi.org/10.1016/S0022-5347(05)64515-6

5. Metcalfe $P D$, Cain $M P$, Kaefer $M$, et al. What is the need for additional bladder surgery after bladder augmentation in childhood? J Urol 2006;176:1801-5. https://doi.org/10.1016/i.juro.2006.03.126

6. MacNeily AE, Jafari S, Scott H, et al. Health-related quality of life in patients with spina bifida: A prospective assessment before and after lower urinary tract reconstruction. J Urol 2009;182:1984-91. https://doi.org/10.1016/i.juro.2009.02.060

7. Liu JS, Dong C, Casey JT, et al. Quality of life related to urinary continence in adult spina bifida patients. Cent Eur J Urol 2015;68:61-7. hrtps://doi.org/10.5173/ceju.2015.01.494

8. Cox A, Breau L, Connor L, et al. Transition of care to an adult spina bifida clinic: Patient perspectives and medical outcomes. J Urol 2011;186:1590-4. https://doi.org/10.1016/i.juro.2011.04.011

9. Higuchi TT, Fox JA, Husmann DA. Annual endoscopy and urine cytology for the surveillance of bladder tumours after enterocystoplasty for congenital bladder anomalies. J Urol 2011;186:1791-5. https://doi.org/10.1016/i.juro.2011.07.028

10. Higuchi $\Pi$ T, Higuchi $\Pi T$, Granberg $C F$, et al. Augmentation cystoplasty and risk of neoplasia: Fact, fiction, and controversy. J Urol 2010;184:2492-6. https://doi.org/10.1016/i.juro.2010.08.038

Correspondence: Dr. Peter Metcalfe, University of Alberta, Edmonton, AB, Canada; pmetcalf@ualberta.ca 